Int. J. Dev. Biol. 55: 505-509

doi: $10.1387 /$ ijdb.103238ct

\title{
Zebrafish embryo, a tool to study tumor angiogenesis
}

\author{
CHIARATOBIA, GIULIA DE SENA and MARCO PRESTA* \\ Department of Biomedical Sciences and Biotechnology, University of Brescia, Italy
}

\begin{abstract}
Zebrafish (Danio rerio) represents a powerful model system in cancer research. Recent observations have shown the possibility to exploit zebrafish to investigate tumor angiogenesis, a pivotal step in cancer progression and target for anti-tumor therapies. Experimental models have been established in zebrafish adults, juveniles, and embryos, each one with its own advantages and disadvantages. Novel genetic tools and high resolution in vivo imaging techniques are also becoming available in zebrafish. It is anticipated that zebrafish will represent an important tool for chemical discovery and gene targeting in tumor angiogenesis. This review focuses on the recently developed tumor angiogenesis models in zebrafish, with particular emphasis to tumor engrafting in zebrafish embryos.
\end{abstract}

KEYWORDS: zebrafish, angiogenesis, xenotransplantation, tumor, VEGF

\section{Introduction}

Angiogenesis plays a key role in tumor growth and metastasis (Carmeliet and Jain, 2000). Thus, the identification of antiangiogenic drugs and of angiogenesis-related targets may have significant implications for the development of anti-neoplastic therapies, as shown by the positive outcomes in the treatment of cancer patients with the monoclonal anti-vascular endothelial growth factor-A (VEGF-A) antibody bevacizumab (Ferrara, 2004).

The teleost zebrafish (Danio rerio) has exceptional utility as a human disease model system and represents a promising alternative model in cancer research (Lam et al., 2006). Zebrafish embryo allows disease-driven drug target identification and in vivovalidation, thus representing an interesting bioassay tool for small molecule testing and dissection of biological pathways alternative to other vertebrate models (Pichler et al., 2003). Indeed, when compared to other vertebrate model systems, zebrafish offers many advantages, including ease of experimentation, drug administration, and amenability to in vivo manipulation. Also, zebrafish is suitable for forward genetic screens and transient or permanent gene inactivation via antisense morpholino oligonucleotide (MO) injection or "targeting-induced local lesions in genes" (TILLING), respectively (Thisse and Zon, 2002). Moreover, the possibility to introduce targeted heritable gene mutations into the zebrafish germ line using engineered zinc-finger nucleases has been reported (Meng et al., 2008). Importantly, zebrafish is suitable for high-throughput screening of chemical compounds using robotic platforms (Funfak et al., 2007; Meng et al., 2008).

Zebrafish possesses a complex circulatory system similar to that of mammals (Weinstein, 2002). The basic vascular plan of the developing zebrafish embryo shows strong similarity to that of other vertebrates (Isogai et al., 2001). At the 13 somite-stage, endothelial cell precursors migrating from the lateral mesoderm originate the zebrafish vasculature and a single blood circulatory loop is present at 24 hours post-fertilization (hpf). Blood vessel development continues during the subsequent days by angiogenic processes. In particular, angiogenesis occurs in the formation of the intersegmental vessels (ISVs) of the trunk that will sprout from the dorsal aorta at $20 \mathrm{hpf}$. Also, subintestinal vein vessels (SIVs) originate from the duct of Cuvier area at $48 \mathrm{hpf}$ and will form a vascular plexus across most of the dorsal-lateral aspect of the yolk ball during the next 24 hours (Isogai et al., 2001).

Various animal models have been developed in rodents and in the chick embryo to investigate the angiogenesis process and for the screening of pro- and anti-angiogenic compounds, each with its own unique characteristics and disadvantages (Hasan et al., 2004). Previous studies had shown that developmental angiogenesis in the zebrafish embryo, leading to the formation of the ISVs of the trunk (Cross et al., 2003) and of the SIV plexus (Serbedzija et al., 2000), represents a target for the screening of anti-angiogenic compounds. In these assays, low molecular weight compounds dissolved in fish water are investigated for their impact on the growth of new blood vessels driven by the complex network

Abbreviations used in this paper: FGF2, fibroblast growth factor-2; hpf, hours postfertilizatio; ISV intersegmental vessel; MO, morpholino oligonucleotide; SIV, subintestinal vein vessel; VEGF, vascular endothelial growth factor.

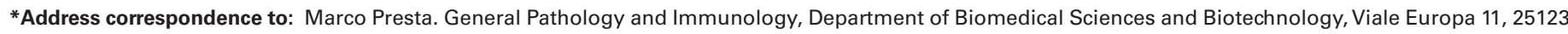
Brescia, Italy. Fax: +39-30-370-1157. e-mail: presta@med.unibs.it
}

Final, author-corrected PDF published online: 27 July 2011.

ISSN: Online 1696-3547, Print 0214-6282

(C) 2011 UBC Press

Printed in Spain 
of endogenous, developmentally regulated signals. Recently, a novel zebrafish yolk membrane (ZFYM) assay has been proposed based on the injection of an angiogenic growth factor [e.g. recombinant fibroblast growth factor-2 (FGF2)] in the perivitelline space of zebrafish embryos in the proximity of developing SIVs. FGF2 induces a rapid and dose-dependent angiogenic response from the SIV basket, characterized by the growth of newly formed, alkaline phosphatase-positive blood vessels (Nicoli et al., 2008a). The ZFYM assay differs from the previous zebrafish-based angiogenesis assays since the angiogenic stimulus is represented by a well-defined, topically delivered exogenous agent that leads to the growth of ectopic blood vessels. This allows the screening of low and high molecular weight antagonists targeting a specific angiogenic growth factor and/or its receptor(s) (Nicoli etal., 2008a).

However, the study of vascular development and on the effects of positive or negative modulators of the embryonic angiogenic process may have important limitations when translated to cancer research. Indeed, tumor-induced vessels show profound morphofunctional alterations when compared to the normal vasculature (Carmeliet and Jain, 2000). This is reflected by significant differences in gene expression profiling between normal and tumorderived endothelium (Ghilardi et al., 2008; St Croix et al., 2000). Thus, the identification of therapeutic targets and the assessment of the efficacy of anti-angiogenic compounds require the development of appropriate animal models in which tumor vasculature can be investigated. To this respect, tumor models have been established in zebrafish embryos, juveniles, and adults [reviewed in (Feitsma and Cuppen, 2008; Stoletov and Klemke, 2008)] that may be suitable for studying the tumor angiogenesis process and its modulators. The availability of imbred, transgenic, gene knockout/knock-in animals, of a wide array of antibodies, as well as of bioinformatic genomic, transcriptomic and proteomic information represent important tools for tumor angiogenesis studies. Several of these tools have been becoming available also for zebrafish.

This review focuses on the recently developed tumor angiogenesis models in zebrafish, with particular emphasis to tumor engrafting in zebrafish embryos.

\section{Angiogenesis in experimental zebrafish tumors}

Zebrafish spontaneously develops almost any type of tumor, most commonly in testis, gut, thyroid, liver, peripheral nerve, connective tissue and ultimobranchial gland. Also, several approaches have been developed to induce cancer in zebrafish. They include treatment with chemical carcinogens, forward genetic screening, target-selected inactivation of tumor suppressor genes, and expression of mammalian oncogenes [see (Feitsma and Cuppen, 2008; Stoletov and Klemke, 2008) for an overview of these approaches and analysis of their main advantages and disadvantages]. Notably, microarray analysis has shown that gene expression signatures are conserved in fish tumors when compared to their human counterpart (Lam et al., 2006).

Noninvasive imaging in zebrafish adults has been attempted. Ultrasound biomicroscopy has been used to follow the growth of liver tumors, their vascularity, and response to treatment (Goessling et al., 2007). Other imaging techniques, including microcomputerized axial tomography, micromagnetic resonance imaging, and optical projection tomography are beginning to be applied in zebrafish and will help to investigate tumor growth and vascularization in adult zebrafish (Spitsbergen, 2007).

Relevant to tumor angiogenesis studies in zebrafish adults, a transparent casper zebrafish line that lacks all types of pigments has been generated (White et al., 2008). Crossing of the casper mutant with transgenic lines that label vasculature or internal organs with fluorescent tags may represent an useful approach to study tumor-host interactions in zebrafish by epifluorescence stereomicroscopy, confocal microscopy, and dual-photon confocal microscopy.

\section{Tumor engrafting and angiogenesis in adult and juvenile zebrafish}

Tumor cell engrafting represents a technique widely used to trigger tumor angiogenesis in recipient animals, mainly rodents (Jensen et al., 2009). Over the past few years, tumor transplantation in zebrafish has developed as an important tumor model (Taylor and Zon, 2009), a major limitation of this approach being the rejection of the tumor graft by the immune system of the host. Relevant to this point, transplantable tumor cell lines have been generated in zebrafish and maintained for several passages in syngeneic and isogeneic adults (Mizgirev and Revskoy, 2006; Mizgirev and Revskoy, 2010). This should allow the study of tumorendothelial cell interactions in immunocompetent zebrafish adults and juveniles. As an alternative approach, immunosuppression by dexamethasone administration (Stoletov et al., 2007) or sublethal $\gamma$ irradiation (White et al., 2008) can be used to prevent the rejection of tumor xenografts (Taylor and Zon, 2009). Also in this case, the use of transparent casper zebrafish may allow the rapid identification of transplanted tumor cells. Indeed, intra-peritoneum or intraventricular injection of small numbers of green fluorescent protein (GFP)-labeled, transgenic zebrafish melanoma cells in irradiated casperrecipients has allowed the study of three-dimensional tumor growth and whole body distribution of tumor cells, thus providing a quantitative analysis of tumor engraftment and offering the potential for monitoring in vivo effects of therapeutically useful molecules (White et al., 2008).

Human cancer cells have been successfully transplanted also in the peritoneal cavity of 30 day-old $T g(f l i 1: E G F P)$ zebrafish juveniles (Stoletov et al., 2007). This has allowed the study of the dynamics of microtumor formation and neovascularization using high resolution imaging techniques, leading to a detailed description of the interaction among fluorescent tumor cells and the GFP-labeled vasculature of the host by three-dimensional reconstruction of confocal microscopy images. The results of these studies have shown that tumor cells secreting human VEGF promote fish vessel remodeling and angiogenesis and that the human metastatic gene $R h o C$ drives the initial steps of the metastatic process. This model system provides a clear window to visualize mechanisms of microtumor formation, cell invasion and tumor-induced angiogenesis in a mature animal. However, due to the fact that juvenile zebrafish has a functional immune system, dexamethasone administration was required to prevent the rejection of the tumor engraftment. Also, at variance with zebrafish embryos (see below), the MO gene targeting approach is unfeasible in zebrafish juveniles. On the other hand, the impact of the tumor graft on the mature vasculature of juvenile fishes may recapitulate more closely the events that occur during tumor angiogenesis in adult animals and cancer patients. Indeed, developing vessels of zebrafish embryos may respond differently 
to tumor grafts compared to the fully developed vasculature of juvenile animals (Stoletov and Klemke, 2008).

\section{Tumor engrafting and angiogenesis in zebrafish embryos}

The optical transparency and ability to survive for 3-4 days without functioning circulation make the zebrafish embryo amenable for vascular biology studies. Also, because of the immaturity of the immune system in zebrafish embryos, no xenograft rejection occurs at this stage (Taylor and Zon, 2009; Weinstein, 2002). Moreover, transient gene inactivation via $\mathrm{MO}$ injection represents a powerful tool for the identification of target genes in zebrafish embryo (Thisse and Zon, 2002).

Original studies have shown the feasibility of injecting human melanoma cells in zebrafish embryos to follow their fate and to study their impact on zebrafish development (Topczewska et al., 2006). In these studies, tumor cells were injected at the blastula
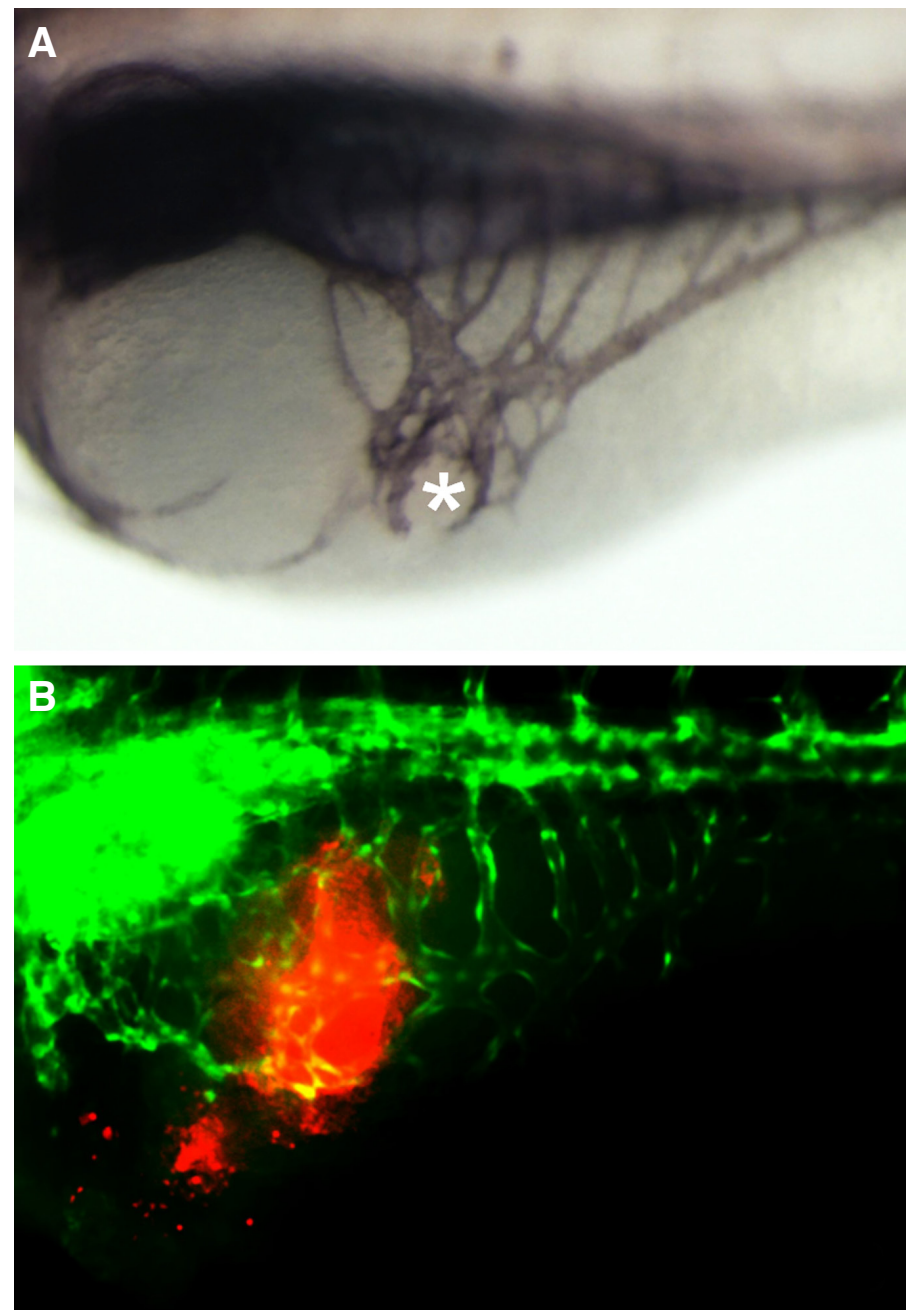

Fig. 1. Tumor angiogenesis in zebrafish embryo. Mammalian tumor cells were injected in the perivitelline space of zebrafish embryos at $48 \mathrm{hpf}$. (A) After 24 hours, embryos were stained for alkaline phosphatase activity to visualize the newly formed blood vessels converging towards the graft (asterisk). (B) GFP-tagged blood neovessels (in green) penetrate the tumor cell graft preloaded with the fluorescent dye Dil (in red) in VEGFR2: G-RCFP transgenic embryos. stage to explore potential bidirectional interactions between cancer cells and embryonic stem cells. The results indicate that developing zebrafish can be used as a biosensor for tumor-derived signals. However, grafting of tumor cells at this stage, well before vascular development, results in their reprogramming toward a non-tumorigenic phenotype, thus hampering any attempt to investigate tumor-driven vascularization. At variance, injection of melanoma cells into the hindbrain ventricle or yolk sac of $48 \mathrm{hpf}$ embryos results in the formation of tumor masses within 4 days (Haldi et al., 2006). Immunostaining analysis of the grafts reveals the presence of blood vessels within the brain and abdominal lesions, even though the high vascularity of the invaded regions may not allow easy discrimination between developmental and tumor-induced angiogenesis (Haldi et al., 2006).

An easier assessment of the angiogenic response triggered by tumor cell engrafting has been obtained by injecting mammalian tumor cells in the proximity of the developing SIV plexus in zebrafish embryos at $48 \mathrm{hpf}$ (Nicoli and Presta, 2007; Nicoli et al., 2007). Pro-angiogenic factors released locally by the tumor graft, including FGF2 and VEGF, affect the normal developmental pattern of the SIVs by stimulating the migration and growth of sprouting vessels towards the implant. One-two days after tumor cell grafting, whole mount phosphatase alkaline staining allows the macroscopic evaluation of the angiogenic response (Fig. 1). The use of transgenic zebrafish embryos, in which endothelial cells express GFP under the control of endothelial-specific promoters [(Baldessari and Mione, 2008) and references therein], represents an improvement of the zebrafish embryo/tumor xenograft model, allowing the observation and time-lapse recording of newly formed blood vessels in live embryos by epifluorescence microscopy as well as by in vivo confocal microscopy (Nicoli and Presta, 2007; Nicoli et al., 2007) (Fig. 1). Also, quantum dots may be used as labeling agents of the zebrafish embryo vasculature for long-lasting intravital time-lapse studies (Rieger et al., 2005).

Hypoxia represents an important driving force for tumor angiogenesis, mainly mediated by VEGF upregulation via activation of the hypoxia inducible factor (HIF) signaling pathway (Pugh and Ratcliffe, 2003). When cancer cells are injected in zebrafish embryos maintained in hypoxic water (7.5\% air saturation), invasion into neighboring tissues, dissemination, and metastasis of labeled tumor cells was greatly enhanced when compared to cells injected under normoxic conditions (Lee et al., 2009). Consistent with increased tumor cell dissemination, hypoxia significantly stimulated neovascularization and tortuosity of the tumor vasculature via tumor cell-derived VEGF upregulation. Accordingly, VEGFR blockade by sunitinib administration in the fish water or by anti-VEGFR2 MO injection inhibited hypoxia-mediated pathological angiogenesis, early dissemination of malignant cells, invasiveness and metastasis.

The possibility to study the metastatic behavior of primary human tumors in zebrafish embryos has been confirmed also when human tumor tissue samples or primary tumor cells are injected into the yolk of 2 day old embryos or are organotopically implanted into the liver of zebrafish larvae (Marques et al., 2009).

\section{Concluding remarks}

When compared to other in vivo tumor angiogenesis assays, the zebrafish embryo/tumor xenograft model presents several advantages. i) The model allows the delivery of a very limited 
number of cells, mimicking the initial stages of tumor angiogenesis and metastasis. ii) Labeled tumor cells (e.g. GFP-transduced or fluorescent dye-loaded cells) can be easily visualized within the embryo. Thus, analysis of the spatial/temporal relationship among tumor cells and newly formed blood vessels may represent an important feature of this model. iii) Several techniques can be applied within the constraints of paraffin or gelatin embedding, including histochemistry and immunohistochemistry. Electron microscopy can also be used in combination with light microscopy. Moreover, reverse transcriptase-polymerase chain reaction analysis with species-specific primers allows the study of gene expression by grafted tumor cells and by the host under different experimental conditions (Nicoli et al., 2007). iv) Zebrafish cancer assays can be scaled-up into medium and high throughput screens (Brittijn et al., 2009; Snaar-Jagalska, 2009).

The identification of genes essential for blood vessel formation is of pivotal importance for the understanding of the angiogenesis process and for the discovery of novel therapeutic targets. In zebrafish embryos, MO injection induce a translational block in gene function (Nasevicius and Ekker, 2000). Gene inactivation by this approach is easy and fast (3-4 days) when compared to the generation of knock-out mice (several months). Also, the simultaneous injection of different MOs may allow the inactivation of more than one gene at the same time. This represents a paramount advantage compared to any mammalian assay available and it can be exploited for the identification of novel gene(s) involved in tumor neovascularization. For instance, MO-induced inactivation of VE-cadherin (Nicoli et al., 2007) or calcitonin receptor-like receptor (Nicoli et al., 2008b) zebrafish gene orthologs results in a significant inhibition of the angiogenic process triggered by the tumor graft in zebrafish embryos. On the other hand, silencing of LIM kinases in human pancreatic cancer cells by siRNA treatment results in a decreased angiogenic potential of these cells when tested in the zebrafish xenograft angiogenesis assay (Vlecken and Bagowski, 2009). Thus, the zebrafish embryo/tumor xenograft model appears to be suitable for the identification of genes involved in the tumor angiogenesis process and expressed either by the host or by tumor cells.

Because of the permeability of its embryos to small molecules, zebrafish allows disease-driven drug target identification and in vivo validation, thus representing an interesting bioassay tool for small molecule testing and dissection of biological pathways alternative to other vertebrate models (Pichler et al., 2003). Accordingly, systemic exposure of live zebrafish embryos to anti-angiogenic compounds dissolved in fish water results in a significant inhibition of neovascularization triggered by the tumor graft (Nicoli etal., 2007; Serbedzija et al., 1999). Also, nanoparticle-mediated targeting of intracellular signaling pathways (Harfouche et al., 2009) and novel anticancer metallodrugs (Brittijn et al., 2009) have been demonstrated to inhibit angiogenesis in this model. Thus, the zebrafish embryo/tumor xenograft model may represent a short-term assay suitable for the identification of novel tumor angiogenesis inhibitors.

It is interesting to note the rapid response of this model to angiogenesis inhibitors (24-48 h) when compared to the chick embryo chorioallantoic membrane assay (3-4 days), the s.c. murine Matrigel plug assay (5-7 days), the murine (1 week) and rabbit (2-3 weeks) cornea assays, and the s.c. mouse syngraft and xenograft assays (several weeks) (Hasan et al., 2004). Also, a large number of zebrafish embryos can be injected and maintained in 96 well- plates, thus allowing systemic in vivo treatment of the animals with minimal amounts of compound. Therefore, dose-response experiments can be easily performed and numerous compounds can be tested in an effective manner. However, the metabolic fate of the drug (either in terms of its activation or inactivation) may differ in zebrafish embryo in respect to mammalian species. Also, zebrafish embryos are maintained at $28^{\circ} \mathrm{C}$. This may not represent an optimal temperature for mammalian cell growth and metabolism, even though we have observed mitotic figures with no sign of apoptosis in grafted tumors throughout the whole experimental period (Nicoli etal., 2007). In this respect, the possibility to raise the incubation temperature up to $35^{\circ} \mathrm{C}$ with no apparent gross effects on zebrafish development has been reported (Haldi et al., 2006).

In conclusion, with its own advantages and disadvantages, the zebrafish embryo/tumor xenograft model represents a novel tool for investigating the neovascularization process exploitable for drug discovery and gene targeting in tumor angiogenesis.

\section{Acknowledgements}

This work was supported by grants from Ministero dell'lstruzione, Università e Ricerca, Associazione Italiana per la Ricerca sul Cancro and Cariplo Foundation.

\section{References}

BALDESSARI, D. and MIONE, M. (2008). How to create the vascular tree? (Latest) help from the zebrafish. Pharmacol Ther 118: 206-230.

BRITTIJN, S.A., DUIVESTEIJN, S.J., BELMAMOUNE, M., BERTENS, L.F., BITTER, W., DE BRUIJN, J.D., CHAMPAGNE, D.L., CUPPEN, E., FLIK, G., VANDENBROUCKE-GRAULS, C.M. et al. (2009). Zebrafish development and regeneration: new tools for biomedical research. Int J Dev Biol 53: 835-850.

CARMELIET, P. and JAIN, R.K. (2000). Angiogenesis in cancer and other diseases. Nature 407: 249-257.

CROSS, L.M., COOK, M.A., LIN, S., CHEN, J.N. and RUBINSTEIN, A.L. (2003). Rapid analysis of angiogenesis drugs in a live fluorescent zebrafish assay. Arterioscler Thromb Vasc Biol 23: 911-912.

FEITSMA, H. and CUPPEN, E. (2008). Zebrafish as a cancer model. Mol Cancer Res 6: 685-94

FERRARA, N. (2004). Vascular endothelial growth factor: basic science and clinical progress. Endocr Rev 25: 581-611.

FUNFAK, A., BROSING, A., BRAND, M. and KOHLER, J.M. (2007). Micro fluid segment technique for screening and development studies on Danio rerio embryos. Lab Chip 7: 1132-1138.

GHILARDI, C., CHIORINO, G., DOSSI, R., NAGY, Z., GIAVAZZI, R. and BANI, M. (2008). Identification of novel vascular markers through gene expression profiling of tumor-derived endothelium. BMC Genomics 9: 201.

GOESSLING, W., NORTH, T.E. and ZON, L.I. (2007). Ultrasound biomicroscopy permits in vivo characterization of zebrafish liver tumors. Nat Methods 4: 551-553.

HALDI, M., TON, C., SENG, W.L. and MCGRATH, P. (2006). Human melanoma cells transplanted into zebrafish proliferate, migrate, produce melanin, form masses and stimulate angiogenesis in zebrafish. Angiogenesis 9: 139-151.

HARFOUCHE, R., BASU, S., SONI, S., HENTSCHEL, D.M., MASHELKAR, R.A. and SENGUPTA, S. (2009). Nanoparticle-mediated targeting of phosphatidylinositol3-kinase signaling inhibits angiogenesis. Angiogenesis 12: 325-338.

HASAN, J., SHNYDER, S.D., BIBBY, M., DOUBLE, J.A., BICKNEL, R. and JAYSON, G.C. (2004). Quantitative angiogenesis assays in vivo-a review. Angiogenesis 7: 1-16.

ISOGAI, S., HORIGUCHI, M. and WEINSTEIN, B.M. (2001). The vascular anatomy of the developing zebrafish: an atlas of embryonic and early larval development. Dev Biol 230: 278-301.

JENSEN, L.D., CAO, R. and CAO, Y. (2009). In vivo angiogenesis and lymphangiogenesis models. Curr Mol Med 9: 982-991.

LAM, S.H., WU, Y.L., VEGA, V.B., MILLER, L.D., SPITSBERGEN, J., TONG, Y., ZHAN, 
H., GOVINDARAJAN, K.R., LEE, S., MATHAVAN, S. et al. (2006). Conservation of gene expression signatures between zebrafish and human liver tumors and tumor progression. Nat Biotechnol 24: 73-75.

LEE, S.L., ROUHI, P., DAHL JENSEN, L., ZHANG, D., JI, H., HAUPTMANN, G., INGHAM, P. and CAO, Y. (2009). Hypoxia-induced pathological angiogenesis mediates tumor cell dissemination, invasion, and metastasis in a zebrafish tumor model. Proc Natl Acad Sci USA 106: 19485-19490.

MARQUES, I.J., WEISS, F.U., VLECKEN, D.H., NITSCHE, C., BAKKERS, J., LAGENDIJK, A.K., PARTECKE, L.I., HEIDECKE, C.D., LERCH, M.M. and BAGOWSKI, C.P. (2009). Metastatic behaviour of primary human tumours in a zebrafish xenotransplantation model. BMC Cancer 9: 128.

MENG, X., NOYES, M.B., ZHU, L.J., LAWSON, N.D. and WOLFE, S.A. (2008). Targeted gene inactivation in zebrafish using engineered zinc-finger nucleases. Nat Biotechnol 26: 695-701.

MIZGIREV, I.V. and REVSKOY, S.Y. (2006). Transplantable tumor lines generated in clonal zebrafish. Cancer Res 66: 3120-3125.

MIZGIREV, I. and REVSKOY, S. (2010). Generation of clonal zebrafish lines and transplantable hepatic tumors. Nat Protoc 5: 383-394.

NASEVICIUS, A. and EKKER, S.C. (2000). Effective targeted gene 'knockdown' in zebrafish. Nat Genet 26: 216-220.

NICOLI, S., DE SENA, G. and PRESTA, M. (2008a). Fibroblast Growth Factor 2-induced angiogenesis in zebrafish: the zebrafish yolk membrane (ZFYM) angiogenesis assay. J Cell Mol Med 13: 2061-2068.

NICOLI, S. and PRESTA, M. (2007). The zebrafish/tumor xenograft angiogenesis assay. Nat Protoc 2: 2918-2923.

NICOLI, S., RIBATTI, D., COTELLI, F. and PRESTA, M. (2007). Mammalian tumor xenografts induce neovascularization in zebrafish embryos. Cancer Res 67: 2927-2931.

NICOLI, S., TOBIA, C., GUALANDI, L., DE SENA, G. and PRESTA, M. (2008b). Calcitonin receptor-like receptor guides arterial differentiation in zebrafish. Blood 111: 4965-4972.

PICHLER, F.B., LAURENSON, S., WILLIAMS, L.C., DODD, A., COPP, B.R. and LOVE, D.R. (2003). Chemical discovery and global gene expression analysis in zebrafish. Nat Biotechnol 21: 879-883.

PUGH, C.W. and RATCLIFFE, P.J. (2003). Regulation of angiogenesis by hypoxia: role of the HIF system. Nat Med 9: 677-684.
RIEGER, S., KULKARNI, R.P., DARCY, D., FRASER, S.E. and KOSTER, R.W. (2005). Quantum dots are powerful multipurpose vital labeling agents in zebrafish embryos. Dev Dyn 234: 670-681.

SERBEDZIJA, G.N., FLYNN, E. and WILLETT, C.E. (1999). Zebrafish angiogenesis: a new model for drug screening. Angiogenesis 3: 353-359.

SERBEDZIJA, G.N., FLYNN, E. and WILLETT, C.E. (2000). Zebrafish angiogenesis: a new model for drug screening. Angiogenesis 3: 353-359.

SNAAR-JAGALSKA, B.E. (2009). ZF-CANCER: developing high-throughput bioassays for human cancers in zebrafish. Zebrafish 6: 441-443.

SPITSBERGEN, J. (2007). Imaging neoplasia in zebrafish. Nat Methods 4: 548-549.

ST CROIX, B., RAGO, C., VELCULESCU, V., TRAVERSO, G., ROMANS, K.E., MONTGOMERY, E., LAL, A., RIGGINS, G.J., LENGAUER, C., VOGELSTEIN, B. et al. (2000). Genes expressed in human tumor endothelium. Science 289: 1197-1202.

STOLETOV, K. and KLEMKE, R. (2008). Catch of the day: zebrafish as a human cancer model. Oncogene 27: 4509-4520.

STOLETOV, K., MONTEL, V., LESTER, R.D., GONIAS, S.L. and KLEMKE, R. (2007). High-resolution imaging of the dynamic tumor cell vascular interface in transparent zebrafish. Proc Natl Acad Sci USA 104: 17406-17411.

TAYLOR, A.M. and ZON, L.I. (2009). Zebrafish tumor assays: the state of transplantation. Zebrafish 6: 339-346.

THISSE, C. and ZON, L.I. (2002). Organogenesis-heart and blood formation from the zebrafish point of view. Science 295: 457-462.

TOPCZEWSKA, J.M., POSTOVIT, L.M., MARGARYAN, N.V., SAM, A., HESS, A.R. WHEATON, W.W., NICKOLOFF, B.J., TOPCZEWSKI, J. and HENDRIX, M.J. (2006). Embryonic and tumorigenic pathways converge via Nodal signaling: role in melanoma aggressiveness. Nat Med 12: 925-932.

VLECKEN, D.H. and BAGOWSKI, C.P. (2009). LIMK1 and LIMK2 are important for metastatic behavior and tumor cell-induced angiogenesis of pancreatic cancer cells. Zebrafish 6: 433-439.

WEINSTEIN, B. (2002). Vascular cell biology in vivo: a new piscine paradigm? Trends Cell Biol 12: 439-445.

WHITE, R.M., SESSA, A., BURKE, C., BOWMAN, T., LEBLANC, J., CEOL, C., BOURQUE, C., DOVEY, M., GOESSLING, W., BURNS, C.E. et al. (2008). Transparent adult zebrafish as a tool for in vivo transplantation analysis. Cell Stem Cell 2: 183-189 


\section{Further Related Reading, published previously in the Int. J. Dev. Biol.}

Insulin-like growth factor-2 regulates early neural and cardiovascular system development in zebrafish embryos Lori Hartnett, Catherine Glynn, Catherine M. Nolan, Maura Grealy and Lucy Byrnes Int. J. Dev. Biol. (2010) 54: 573-583

Reprogramming of melanoma cells by embryonic microenvironments Alejandro Díez-Torre, Ricardo Andrade, Cristina Eguizábal, Elixabete López, Jon Arluzea, Margarita Silió and Juan Aréchaga Int. J. Dev. Biol. (2009) 53: 1563-1568

Zebrafish development and regeneration: new tools for biomedical research Sebastiaan A. Brittijn, Suzanne J. Duivesteijn, Mounia Belmamoune, Laura F.M.Bertens, Wilbert Bitter, Joost D. de Bruijn, Danielle L. Champagne, Edwin Cuppen, Gert Flik, Christina M. Vandenbroucke-Grauls, Richard A.J. Janssen, Ilse M.L. de Jong, Edo Ronald de Kloet, Alexander Kros, Annemarie H. Meijer, Juriaan R. Metz, Astrid M. van der Sar, Marcel J.M. Schaaf, Stefan Schulte-Merker, Herman P. Spaink, Paul P. Tak, Fons J. Verbeek, Margriet J. Vervoordeldonk, Freek J. Vonk, Frans Witte, Huipin Yuan and Michael K. Richardson Int. J. Dev. Biol. (2009) 53: 835-850

Vasculogenesis and angiogenesis in the mouse embryo studied using quail/mouse chimeras Michel Pudliszewski and Luc Pardanaud Int. J. Dev. Biol. (2005) 49: 355-361

Large scale genetics in a small vertebrate, the zebrafish.

$P$ Haffter and C Nüsslein-Volhard

Int. J. Dev. Biol. (1996) 40: 221-227

The chick embryo chorioallantoic membrane as a model for in vivo research on angiogenesis. D Ribatti, A Vacca, L Roncali and F Dammacco Int. J. Dev. Biol. (1996) 40: 1189-1197

5 yr ISI Impact Factor $(2009)=3.253$

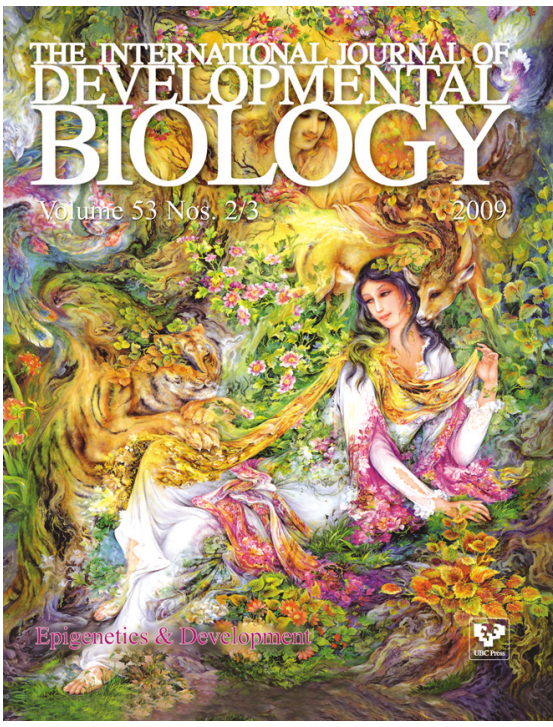

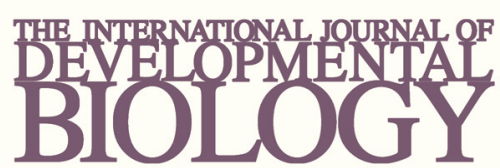

Volume 54 Nos. 6/7
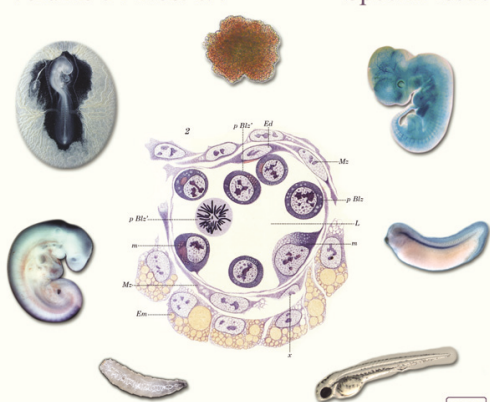

Developmental Hematopoiesis

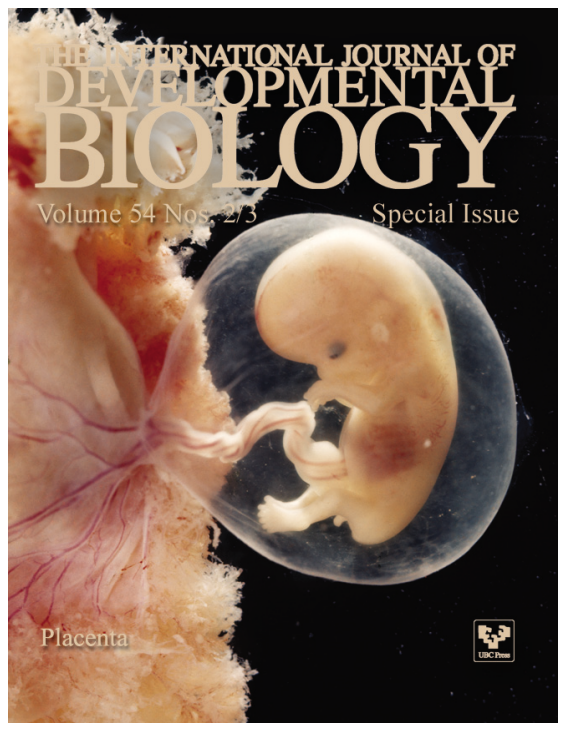

